\title{
Design of Travelling Wave Relay for Protection of Transmission Lines
}

\author{
Modawy Adam Ali Abdalla ${ }^{1}$, Giddani Kalcon ${ }^{2}$, Ahmed Mohammed $^{3}$ \\ Department of Electrical Engineering, Sudan University of science and Technology, Khartoum, Sudan \\ *Corresponding Author: Modawy Adam Ali Abdalla, Department of Electrical Engineering, Sudan \\ University of science and Technology, Khartoum, Sudan
}

\begin{abstract}
Travelling wave relay operation is based on propagated forward and backward electromagnetic waves initiated when short circuit occur in transmission line. The travelling wave's information's could be used to calculate the line impedance and signal the $C B$ in faulty conditions. In this paper, traveling wave numeric relay based on analog signal processing is proposed to identify and locate the fault by using the information contained in the waves. The techniques are implemented in MATLABSIMULINK. Some cases of the fault are discussed in this paper
\end{abstract}

Keywords: traveling waves, transmission line, High Voltage (HV), tripping signal,

\section{INTRODUCTION}

The rapid and dramatically growth of electric power systems over the past few decades has resulted in a large increase of the number of lines in operation and their total length. These lines are exposed to faults as a result of lightning, short circuits, faulty equipment, human errors, overload, and aging. Severe damage may take place if faults are not eliminated as fast as possible and may leads to total black-out in the system.

Accurate and proper protection system is very important in order to eliminate the faulty section of the transmission lines [2]. Devices, called protective relays, are installed at various places in the power system to detect faults and isolate the faulted part from the remaining system. Nowadays, fast travelling wave relays is replacing the traditional relays based on static and digital devices in EHV transmission system. Traveling eave relay reduced the fault clearing time, determine the location of the fault and reduce the damages and increase safety standard. Traveling wave phenomenon in high voltage transmission lines constitutes one of the shortest system transients. Traveling waves are associated with the propagation of electromagnetic waves which result from the short circuits in transmission lines and the lightning or switching operations in power system.

A sudden and significant change in voltage in at least one place within the high voltage line leads to the initiation of an electromagnetic wave which propagates from that point in opposite directions.

Electromagnetic wave can be divided into a voltage wave and a current wave associated with the magnetic field moving along the line with finite speed.

This paper investigates the operation of numeric relay based on travelling wave algorithm. The travelling wave algorithm is based on impedance calculation, voltage and current measurement. The study is carried-out in SIMULINK environment.

\section{Traveling WaVes Theory}

The disturbance of the transmission line at any point is appears as a change in steady state power equation and propagate travelling wave in both directions of line terminals.

Consider a small section of length dx of transmission line as shown in Figure (1) travelling wave of the voltage (V) and current (I) are generated when a fault occurs on the transmission line. 


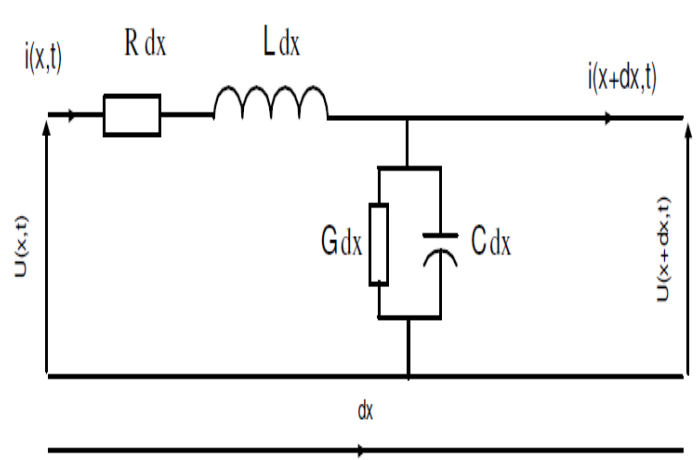

Figure1. Single-phase transmission line

The travelling waves of voltage drop in the positive $\mathrm{x}$ direction in the magnetic flux crated by the electromagnetic wave.

$\frac{d V}{d x}=R i+L \frac{d i}{d t}$

The travelling wave of the current through the leakage conductance and capacitance

$\frac{d i}{d x}=V G+C \frac{d V}{d t}$

Taking Laplace transforms with respect to time variable $t$ for equation (1) and (2) and using he general solution of this equation we found

$V_{x}=\left(V_{F}+\frac{I_{F} Z_{c}}{2}\right) e^{\alpha x} e^{j(\omega t+\beta x)}+\left(V_{F}-\frac{I_{F} Z_{c}}{2}\right) e^{-\alpha x} e^{-j(\omega t+\beta x)}$

$I_{x}=\left(\left(V_{F} / Z_{c}+I_{F}\right) / 2\right) \mathrm{e}^{\alpha \mathrm{x}} \mathrm{e}^{\mathrm{j}(\mathrm{wt}+\beta \mathrm{x})}-\left(\left(V_{F} / Z_{c}-I_{F}\right) / 2\right) \mathrm{e}^{-\alpha \mathrm{x}} \mathrm{e}^{-\mathrm{j}(\mathrm{wt}+\beta \mathrm{x})}$

Equation (3) and (4) it is used to obtained travelling wave at any point on the line at distance $\mathrm{X}$ from the fault point. Where

$V_{F}$ And $I_{F}$ it is post fault voltage and current

$\alpha=$ attenuation constant

$\beta=$ phase constant

\subsection{Forward and Backward of the Travelling Waves}

The propagated travelling wave consists of two terms as shown in Figure. 2 and equations (3) and (4). The first one progresses along the line from the sending end toward the receiving end. And the second term diminishes in magnitude and is retarded in phase from the receiving end toward the sending end.

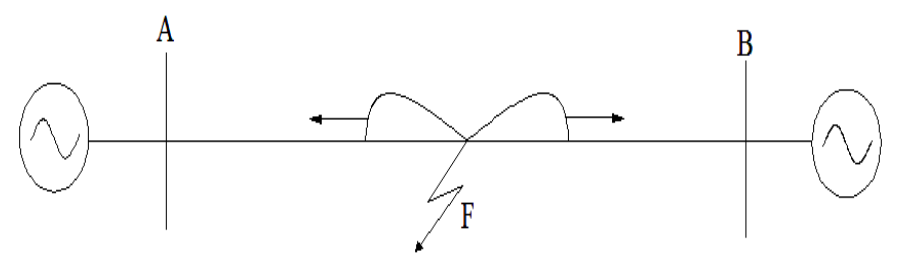

Figure2. Propagation of electromagnetic wave as a result of fault

The two terms as function of two variables time $(\mathrm{t})$ and distance $(\mathrm{X})$

$\mathrm{V}_{\mathrm{x}}=\mathrm{V}^{\mathrm{f}}+\mathrm{V}^{\mathrm{r}}$

Where

$V^{f}=\left(\left(V_{F}+Z_{c} I_{F}\right) / 2\right) e^{\alpha x} e^{j(w t+\beta x)}$

it is called forward travelling voltage wave.

$V^{r}=\left(\left(V_{F}-Z_{c} I_{F}\right) / 2\right) e^{-\alpha x} e^{-j(w t+\beta x)}$ 
it is called reflected voltage or reverse travelling voltage wave.

And it is similarly the current travelling wave equation.

$I_{x}=I^{f}-I^{r}$

Where

$I^{f}=\left(\left(V_{F} / Z_{c}+I_{F}\right) / 2\right) e^{\alpha x} e^{j(w t+\beta x)}$

it is called forward travelling current wave.

$I^{r}=\left(\left(V_{F} / Z_{c}-I_{F}\right) / 2\right) e^{-\alpha x} e^{-j(w t+\beta x)}$

\section{Reflection and Refraction of Traveling Waves}

Traveling waves travel along the transmission line and encounter discontinuities, such as buses and transformers. When traveling waves reach a discontinuity, part of it is reflected back and the remaining part passes through. The magnitude of the reflected and refracted waves depends on the characteristic impedance of the transmission line and the impedance beyond the discontinuity. The amplitude of the reflected and refracted waves is such that the proportionality of the voltage and current is preserved. The phenomenon of the reflection and refraction of traveling waves is shown in the Bewley's Lattice diagram, which is reproduced in Figure 3 This diagram shows the propagation of traveling waves that originates at a fault location that is $80 \mathrm{~km}$ from bus A, on a transmission line of $100 \mathrm{~km}$ length.

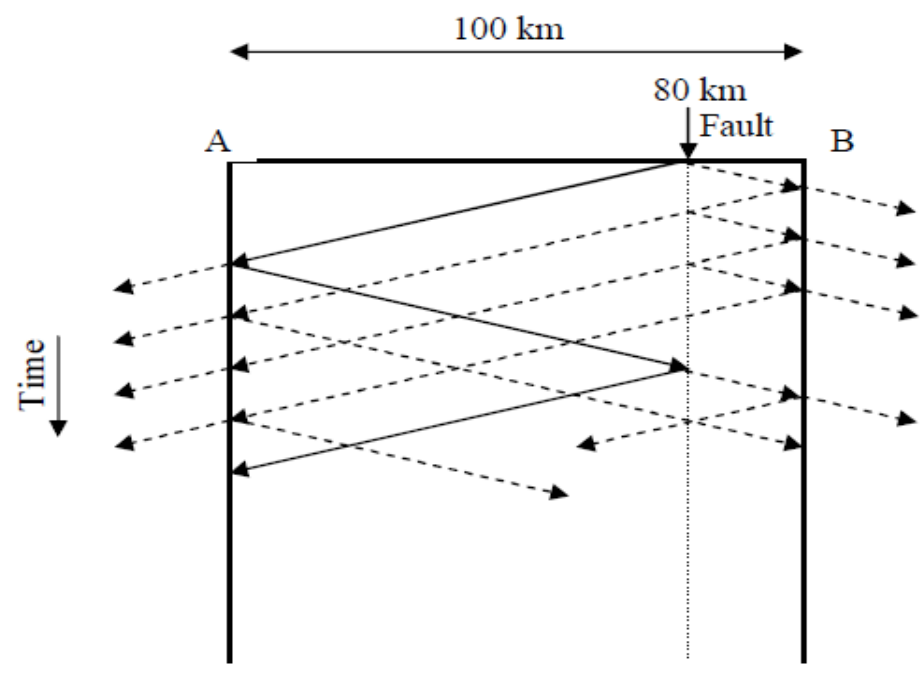

Figure3. Bewley's Lattice diagram

At each discontinuity, the total energy of the incident wave is distributed among the reflected and refracted waves. This process lasts until the traveling waves loose all their energy and their amplitudes become negligible.

This ratio, called the voltage reflection factor, $\rho_{\mathrm{V}}$, is given by

$\rho_{V}=\frac{Z_{t}-Z_{0}}{Z_{t}+Z_{0}}$

Similarly, the current reflection factor, $\rho_{\mathrm{i}}$, is given by

$\rho_{i}=\frac{Z_{0}-Z_{t}}{Z_{0}+Z_{t}}$

\subsection{Traveling Wave Relays}

Several traveling wave relays have been proposed in the past, but all of them use analog technology. Due to the limitations in detecting high frequency waves, these techniques have not been used in commercial devices.

The basic concept of previously proposed techniques is presented in this section. A fault on a transmission line can be replaced by a fictitious source as shown in Figure 4. Let the voltage and current injected at the fault be $V_{f}$ and $I_{f}$. These injected signals can be calculated by subtracting the 
pre-fault voltage and current from the post fault voltage and current. Fault injected components; therefore, can be expressed in terms of the forward and backward traveling waves as

$V_{f}(x, t)=f^{+}\left[t-\frac{x}{v}\right]+f^{-}\left[t+\frac{x}{v}\right]$

$i_{f}(x, t)=\frac{1}{Z_{0}}\left[f^{+}\left[t-\frac{x}{v}\right]-f^{-}\left[t+\frac{x}{v}\right]\right]$

Where,

$f^{+}$is a function representing the forward traveling wave.

$f^{-}$is a function representing the backward traveling wave.

$v$ is velocity of propagation of traveling waves.

$Z_{0}$ is surge impedance of the transmission line, and

$x$ is the distance traveled by the traveling waves.

Rearranging Equations 13 and 14 provides,

$$
\begin{aligned}
& 2 f^{+}\left[t-\frac{x}{v}\right]=V_{f}(x, t)+Z_{0} i_{f}(x, t) \\
& 2 f^{-}\left[t+\frac{x}{v}\right]=V_{f}(x, t)-Z_{0} i_{f}(x, t)
\end{aligned}
$$

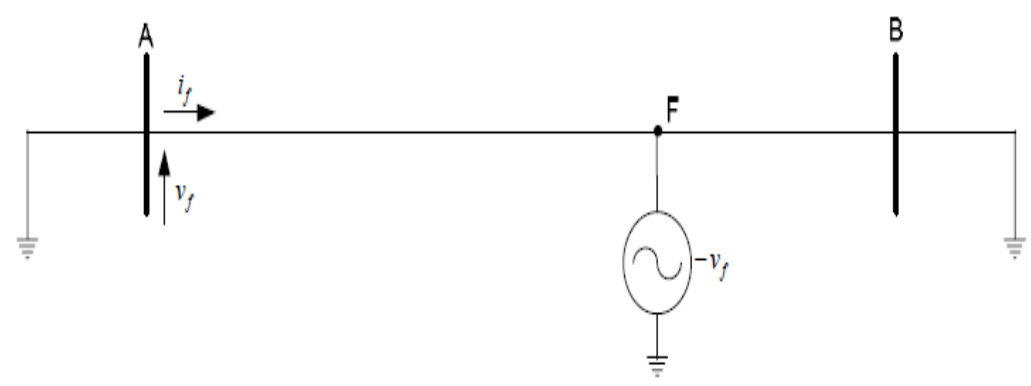

Figure4. Voltage and current components injected by the fictitious source at the fault

\section{Design of Numeric TRAVELling WAVES Relay}

Numerical protection relays operate on the basis of sampling inputs and controlling outputs to protect or control the monitored system. System currents and/or voltages, for example, are not monitored on a continuous basis but, like all other quantities, are sampled one at a time. After acquiring samples of the input waveforms, calculations are performed to convert the incremental sampled values into a final value that represents the associated input quantity based on a defined algorithm. Once the final value of an input quantity can be established, the appropriate comparison to a setting, or reference value, or some other action, can be taken as necessary by the protection relay. Depending upon the algorithm used, and other system design or protection requirements, the final value may be calculated many times within a single sampling cycle, or only once over many cycles.

\section{Design of Travelling Wave Relay}

The numeric travelling wave relays include hardware and software components.

\subsection{Hardware Components}

The Hardware component used in travelling wave relay it is consist from group of element voltage transformer (VT) and current transformer (CT) as isolated transformer, All the signals passed through filter, Sample and Hold S\&H, Analogue to digital convertor A/D, analogue multiplexer, algorithm implemented in travelling wave equation, and Decision.

\subsection{Proposed Protection Scheme}

the decision making process in proposed transient based protection scheme and overall functionality of the numerical relay model used in such scheme are explained. The model for a transient based numerical relay unit installed at each terminal of the transmission line is proposed in Figure 5. 


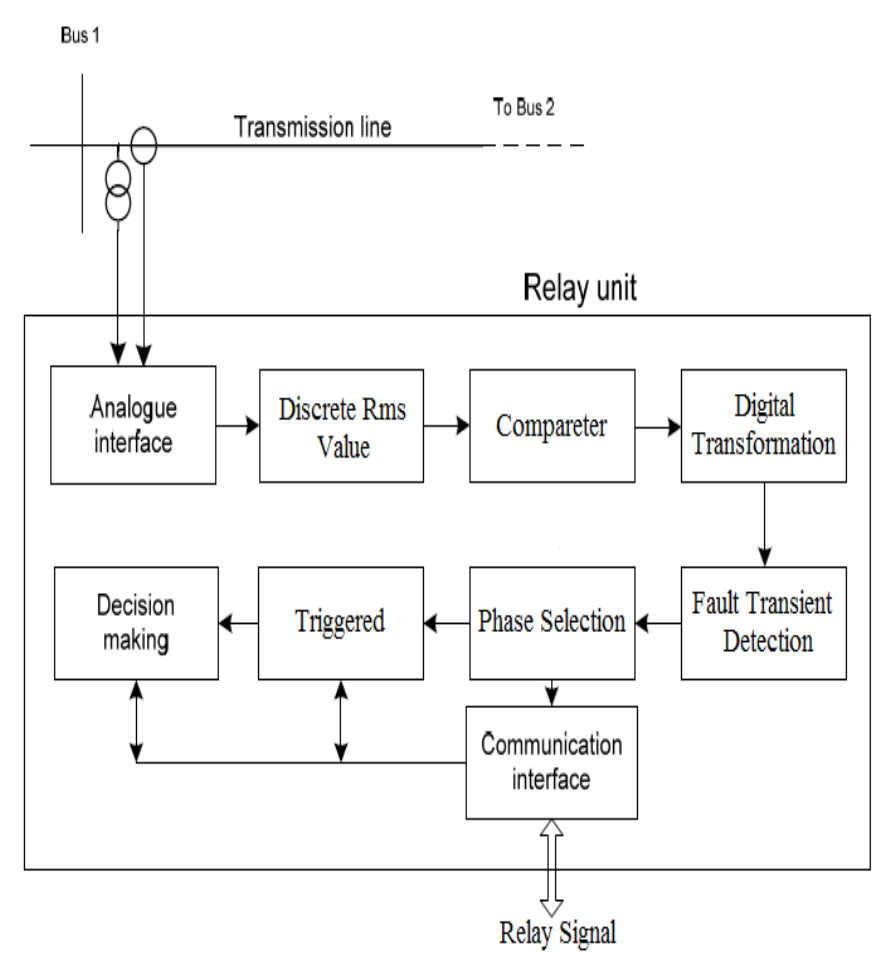

Figure5. Transient based numerical relay unit

\subsection{Developed algorithm}

The traveling wave based protection algorithms are usually based on analysis of measured transients in voltage and current quantities at transmission line terminals. The traveling waves propagated in the system due to fault inceptions and we find Implementation of the Algorithm in the figure (6)

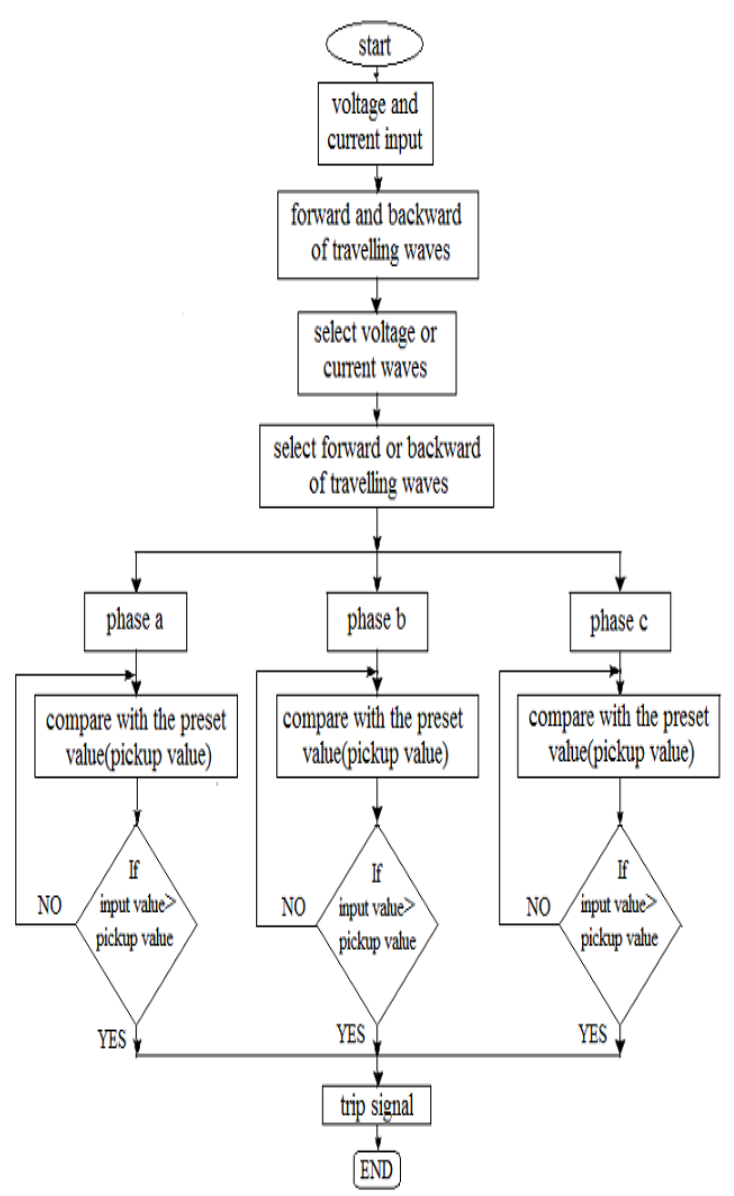

Figure6. Travelling Wave Algorithm 


\subsection{Simulink Diagram of Trevelling Wave Relay}

The Simulink design to simulate travelling wave relay is show in figure 7 travelling wave calculating block is built using equation(9) to equation (16) the output of travelling wave block forward current (if) passing through discrete (RMS) each phase it is comparing to setting value through (AND) gate and using triggered as step function finally using (XOR) gate as trip signal.

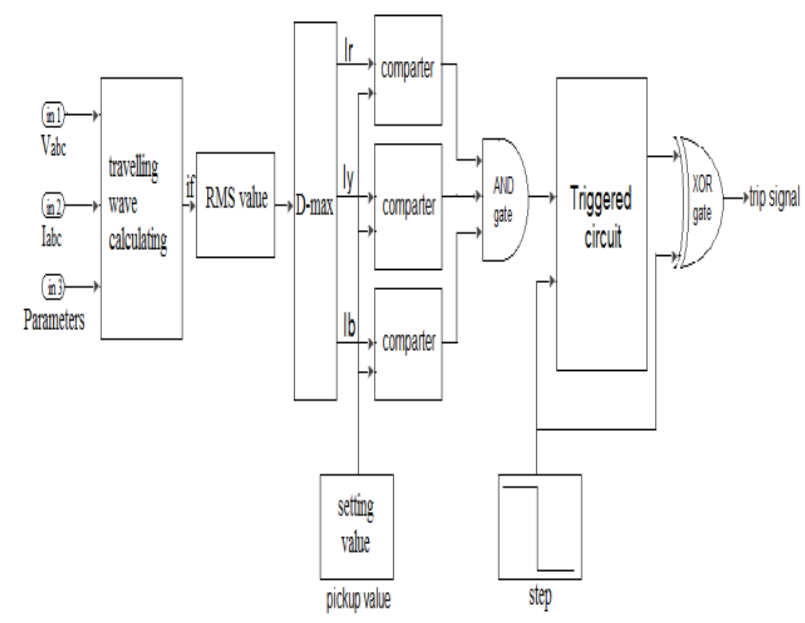

Figure7. Travelling wave relay Implemented in MATLAB/Simulink

\section{Simulation Results}

This section presents the simulation results to evaluate the operation of developed travelling wave relay. The developed travelling wave relay is implemented in Matlab Simulink environment. The model has been created with a toolbox of Matlab/Simulink called Sim Power Systems. It is a collection of blocks that allow the modeling of different elements that usually are present on power systems.

\subsection{System Lay-Out}

To study the operation of traveling waves relay, the system in figure 8 is built in Simulink environment. The proposed system is composes of three-phase voltage source supplies a static load via $\pi$-section transmission line. Travelling waves relay is located at beginning of the line to protect the line against abnormal conditions. The transmission line under study is operating at $400 \mathrm{kV}$, $1200 \mathrm{MVA}$ short-circuit level. A load of $400 \mathrm{kV}, 100 \mathrm{MW}$ and $100 \mathrm{MVAr}$ rating is connected at the sending end $B_{2}$ through transmission line of $200 \mathrm{~km}$ long connected between $B_{1}$ and $B_{2}$.

Table1. Simulation model software with following parameters

\begin{tabular}{|c|c|}
\hline Component & Parameters \\
\hline Voltage source & $400 \mathrm{KV}, 60 \mathrm{~Hz}, 1200 \mathrm{MVA}, \mathrm{X} / \mathrm{R}=8$ \\
\hline Transmission Line & $200 \mathrm{~km}, 400 \mathrm{KV}, \mathrm{R}_{1}=0.01273 \Omega / \mathrm{Km}, \mathrm{L}_{1}=0.9337 \mathrm{e}-$ \\
& $3 \mathrm{H} / \mathrm{Km}, \mathrm{C}_{1}=12.74 \mathrm{e}-9 \mathrm{~F} / \mathrm{Km}, \mathrm{R}_{0}=0.3864 \Omega / \mathrm{Km}$, \\
$\mathrm{L}_{0}=4.126 \mathrm{e}-3 \mathrm{H} / \mathrm{Km}, \mathrm{C}_{0}=7.751 \mathrm{e}-9 \mathrm{~F} / \mathrm{Km}$ \\
\hline Fault resistance & $100 \mathrm{ohm}$ \\
\hline
\end{tabular}

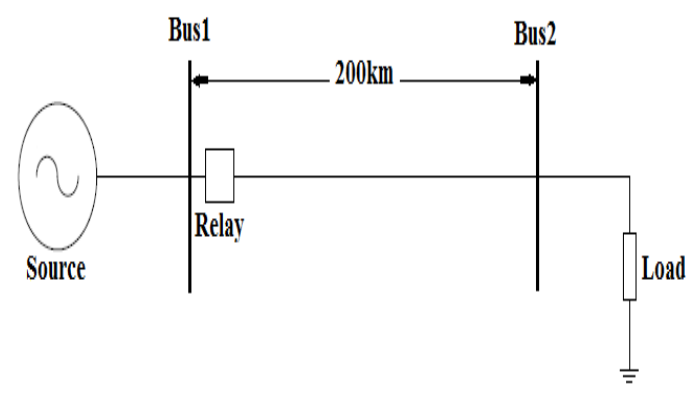

Figure8. Single line diagram of Power System under Study 


\subsection{Simulation Cases Results}

The investigation of the travelling waves relay is carried out using Matlab Simulink environment. The system in Fig.4.1 is modeled and simulated in the matlab software with the parameters given in table 4.1.

The operational scenarios for traveling wave relay investigation include:

a) Operational during normal condition

b) Operational when symmetrical fault occur in the mid of transmission line

c) Operational when unsymmetrical fault occur in the mid of transmission line

\subsubsection{Investigations during normal operation}

The system in Figure 8 is operated in normal operation condition without any disturbance for period of $0.2 \mathrm{~s}$. The results obtained simulation is shown in figure 9. As shown in figure $9 \mathrm{a}$ and figure $9 \mathrm{~b}$ the waveforms of the voltages and current are uniform during simulation period without any disturbances. Also, the travelling wave components (forward and backward currents) calculated by the travelling wave relay are uniform during simulation period as shown in figure $9 \mathrm{c}$ and figure $9 \mathrm{~d}$. The relay signal sent to circuit break in this case is 1 which mean the breakers should close its contacts to allow the current to flow in the system and supplies the static load.

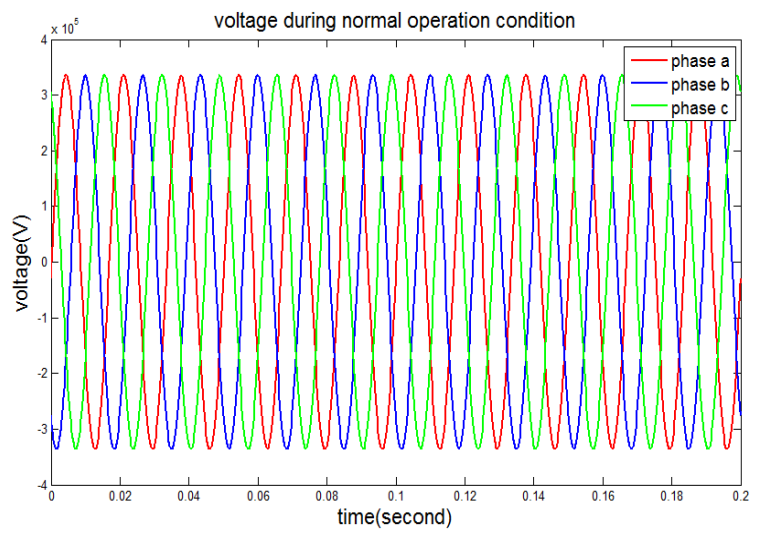

Figure9a. System voltage wave during normal operation

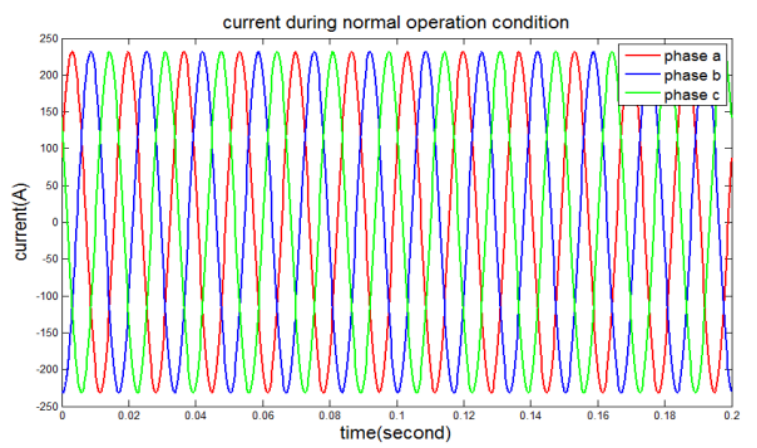

Figure9b. System current wave during normal operation

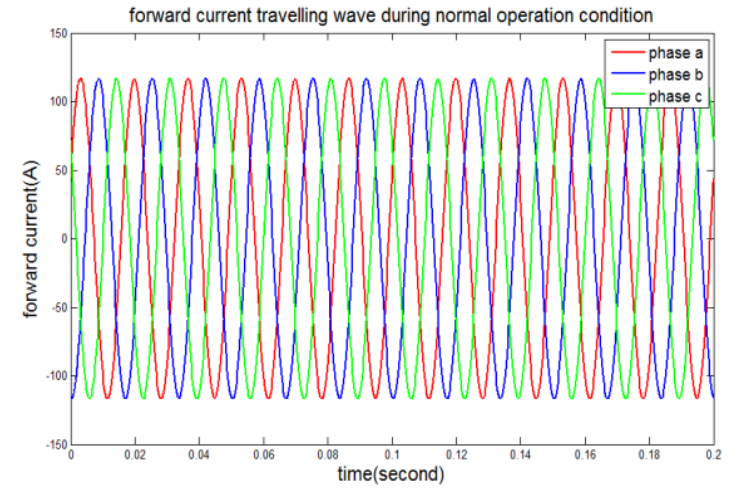

Figure9c. Forward travelling wave during normal operation 


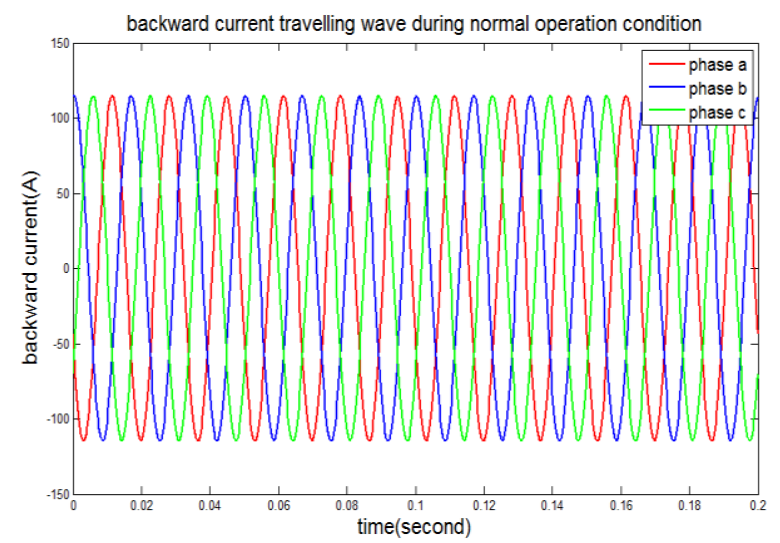

Figure9d. Backward travelling wave during normal operation

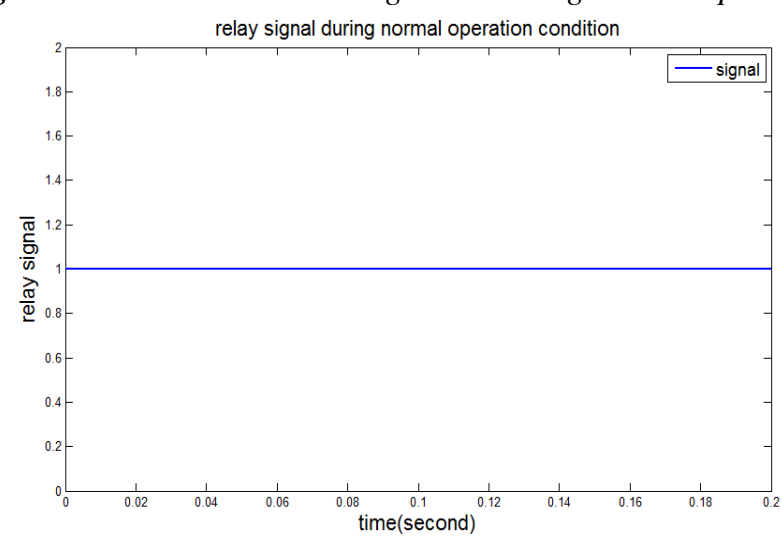

Figure9e. Relay signal during normal operation

\subsubsection{Investigations during symmetrical fault}

To demonstrate the ability of travelling wave relay during abnormal conditions, the system in figure 8 is subjected to three phase symmetrical fault in the middle of transmission line for duration of $0.1 \mathrm{sec}$. The results obtained during the simulation are shown in figure 10. As shown in figure 10a and figure $10 \mathrm{~b}$ the waveforms of the voltages and current observe increases in current and decrease in voltage when the fault occur and recover again when fault is cleared. The system is subjected to 6 times the rated current for duration of $0.1 \mathrm{~s}$ and this may destroy the entire system. If travelling wave relay is used for protection of the line than the system is subjected to high current for duration of just $0.01 \mathrm{~s}$ until the breaker eliminate the fault as shown in figure 10d. The travelling wave components (forward and backward currents) calculated by the relay are ununiformed during simulation period as shown in figure $10 \mathrm{c}$ and figure $10 \mathrm{~d}$ which indicate occurring of abnormal condition and initiate the relay to send trip signal to the breaker just after appearance of the fault as shown in figure 10e. The relay signal to circuit break in this case is change from 1 to 0 at $0.05 \mathrm{~s}$ which mean the breakers should open its contact and clear the fault. Fig4.3f and Fig4.3g showed the voltage and current waveform after operation of breaker. The voltage is subjected to transient decrease but recover to nominal values while the current is fall to zero because the breakers are open.

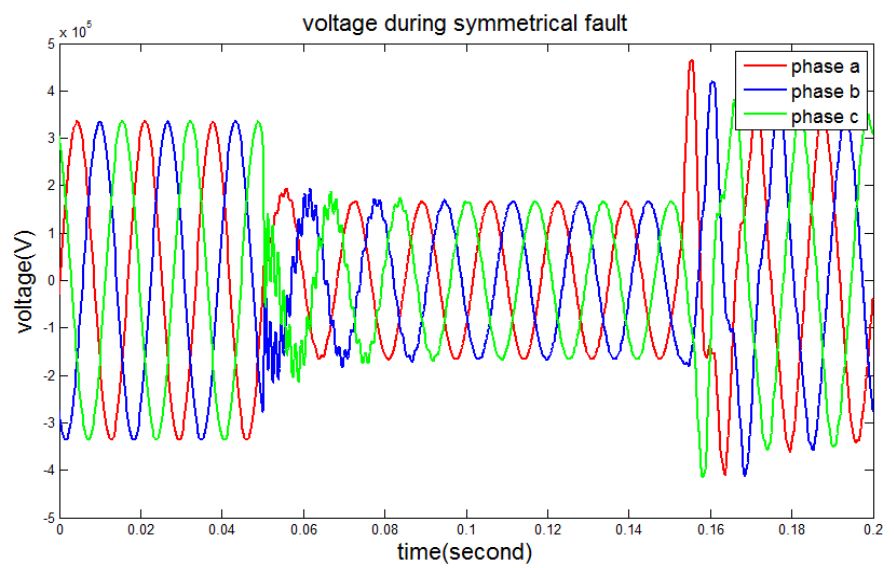

Figure10a. System voltage wave during symmetrical fault 


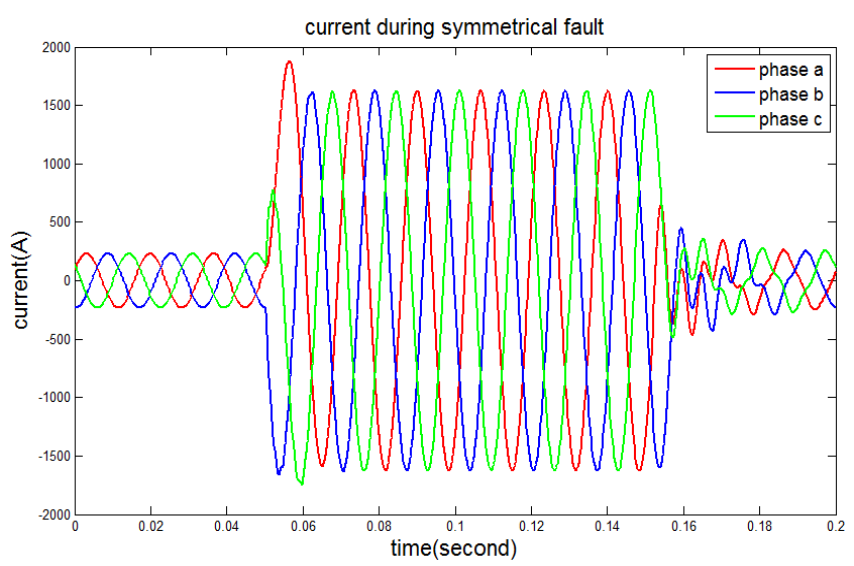

Figure10b. System current wave during symmetrical fault

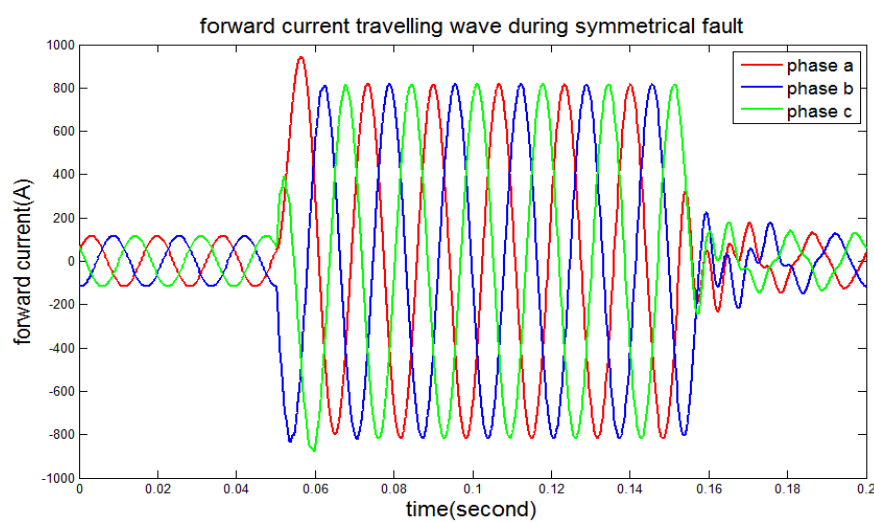

Figure10c. Forward travelling wave during symmetrical fault

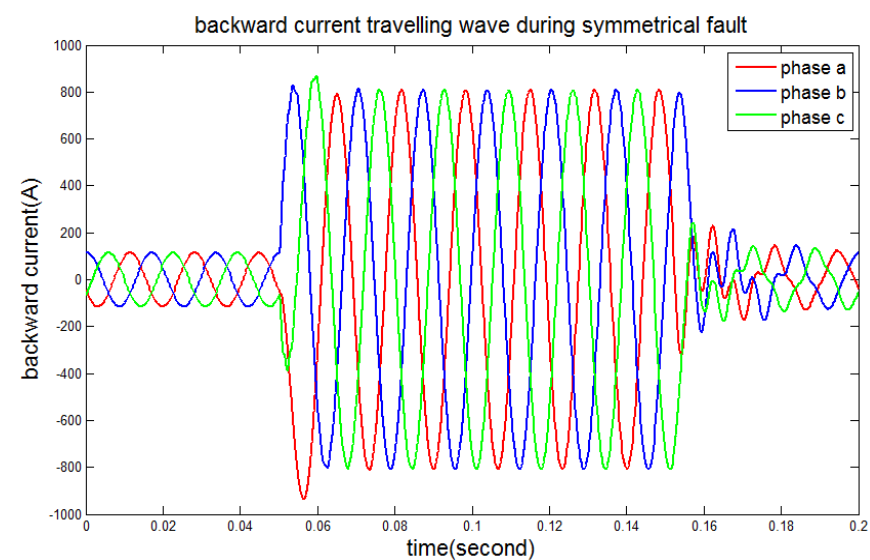

Figure10d. Backward travelling wave during symmetrical fault

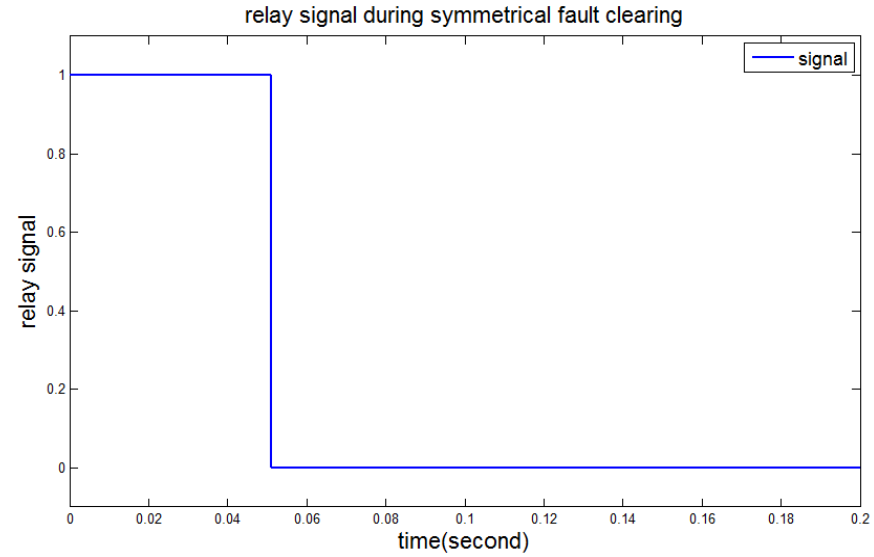

Figure10e. Relay signal during symmetrical fault clearing 


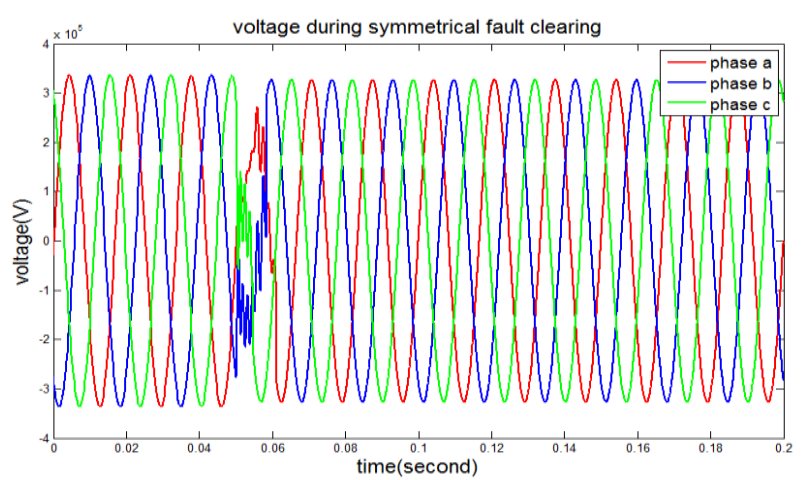

Figure10f. System voltage wave during symmetrical fault clearing

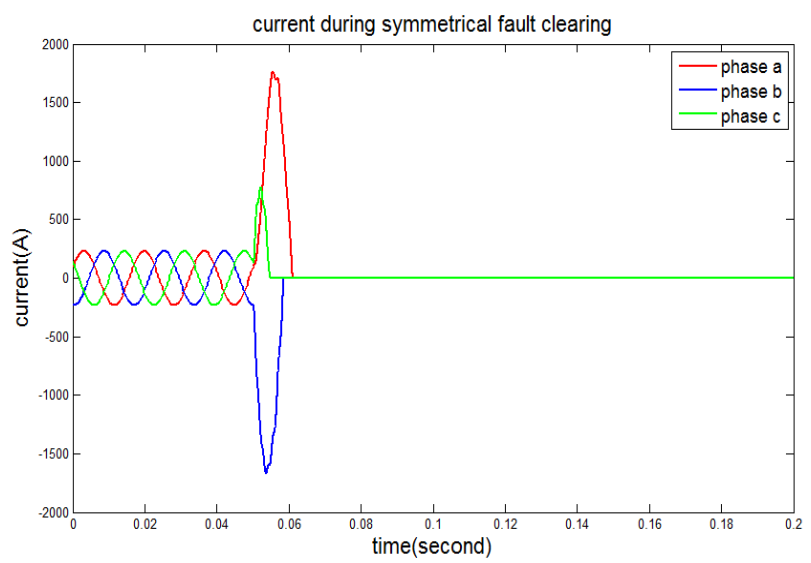

Figure10g. System current wave during symmetrical fault clearing

\subsubsection{Investigations During Unsymmetrical Fault}

The system in figure 8 is subjected to phase to ground unsymmetrical fault in the middle of transmission line for duration of $0.1 \mathrm{sec}$. figure 11 show the simulation result during occurring of phase to ground fault. As shown in figure 11a the voltage of phase a is decrease during fault period and recover again when the fault is eliminated. Also phase a current is increase dramatically to about 6 times the rated value and this will destroy the system components if no proper protection is used as shown in figure 11b. Again if travelling wave relay is used for protection of the line than the system is subjected to high current for duration of just $0.01 \mathrm{~s}$ until the breaker eliminate the fault as shown in figure 11g. The travelling wave components regarding phase a (forward and backward currents) calculated by the relay are ununiformed during simulation period as shown in figure 11c and figure $11 \mathrm{~d}$ which indicate occurring of abnormal condition and initiate the relay to send trip signal to the breaker just after appearance of the fault as shown in figure 11e. The relay signal to circuit break in this case is change from 1 to 0 at $0.05 \mathrm{~s}$ which mean the breakers should open its contact and clear the fault.

figure $11 \mathrm{f}$ and figure $11 \mathrm{~g}$ showed the voltage and current waveform after operation of breaker. The voltage is subjected to transient decrease but recover to nominal values while the current is fall to zero because the breakers are open.

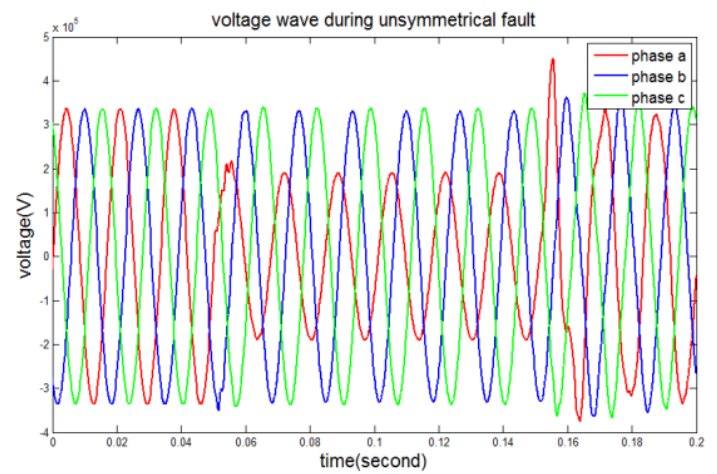

Figure11a. System voltage wave during unsymmetrical fault 


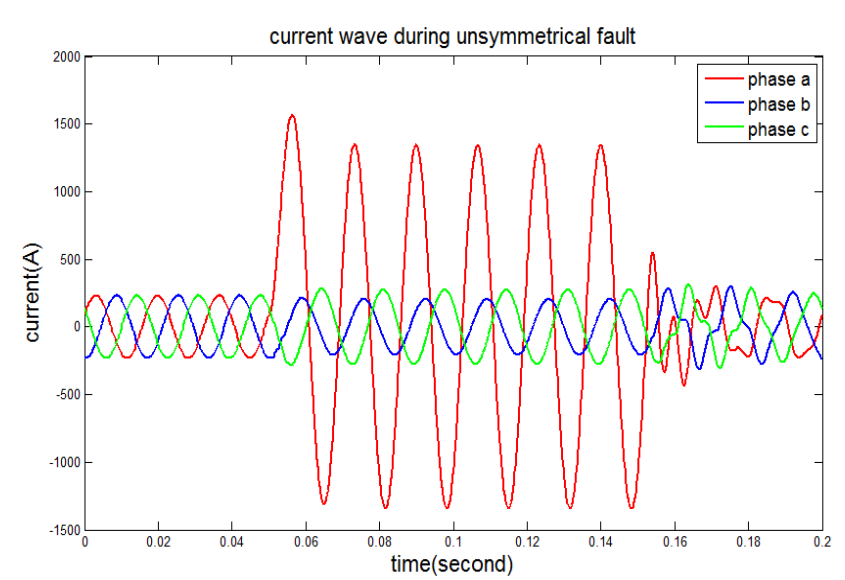

Figure11b. System current wave during unsymmetrical fault

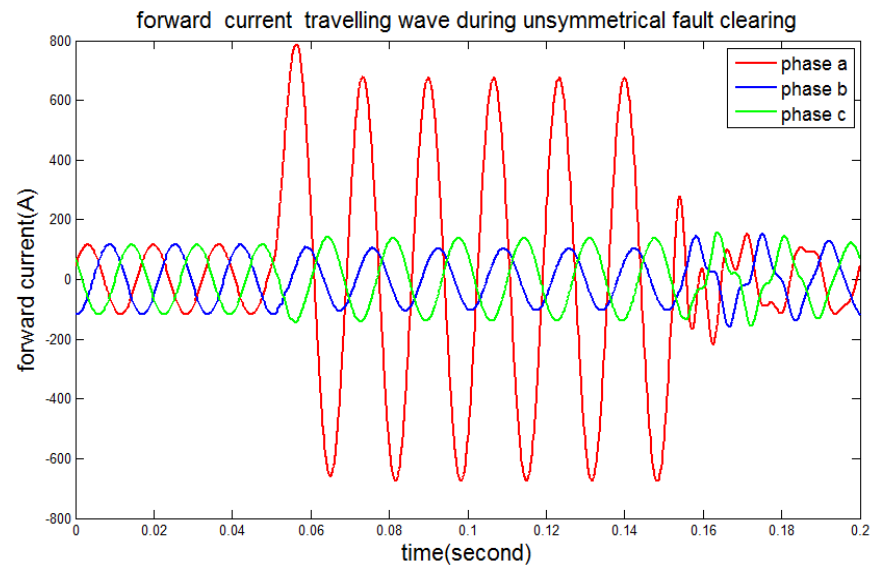

Figure11c. Forward travelling wave during unsymmetrical fault

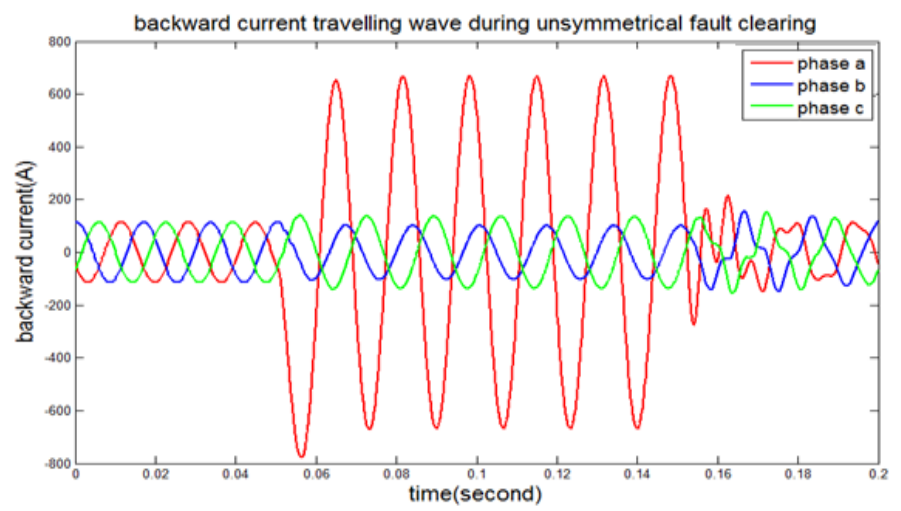

Figure11d. Backward travelling wave during unsymmetrical fault

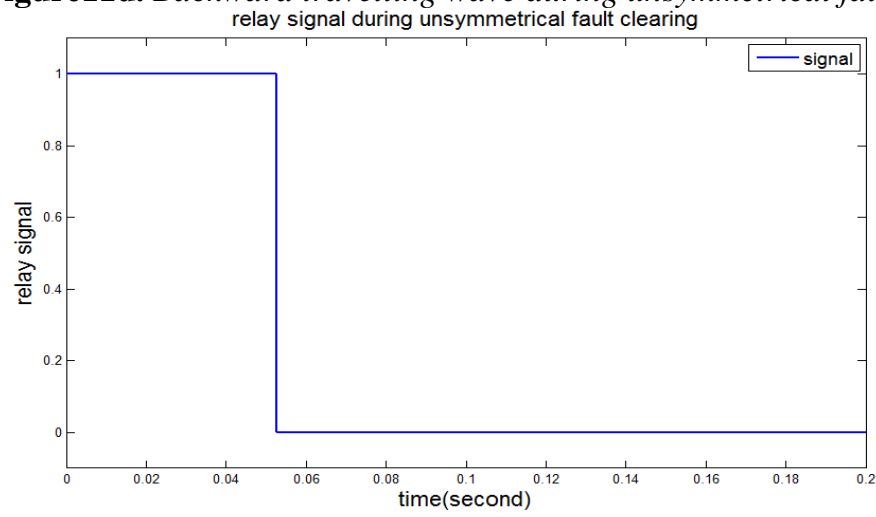

Figure11e. Relay signal during unsymmetrical fault clearing 


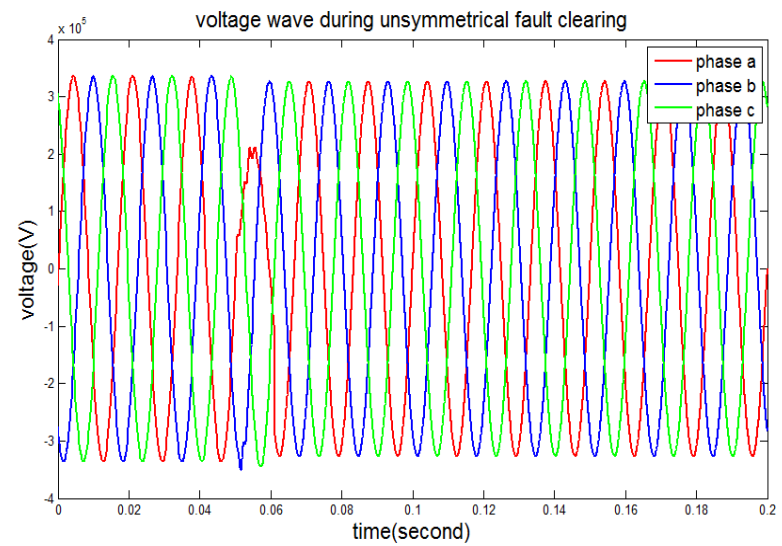

Figure11f. System voltage wave during unsymmetrical fault clearing

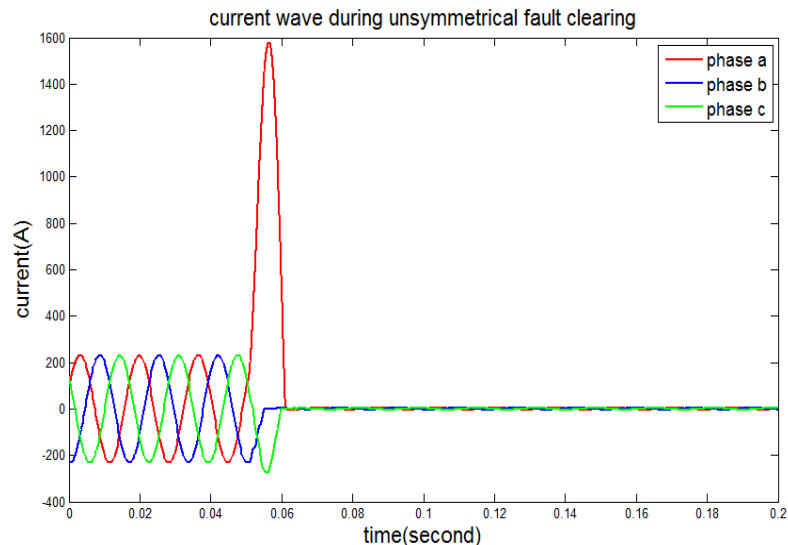

Figure11g. System current wave during unsymmetrical fault clearing

\section{CONCLuSion}

The protection is needed to remove as speedily as possible any element of the power system in which a fault has developed so long as the fault remains connected. Fault on the transmission line needs to be restored as quickly as possible. The sooner it is restored, the less the risk of power outage, damage in equipment of grid. The proposed relay is based on travelling waves generated when fault occur in the line. The travelled wave components in opposite sides can be used to determine the line states and the location of the fault if fault is happened. The developed algorithm is equipped in the microprocessor of numeric relay with proper software and hardwiring. The methodology based on travelling wave was developed and tested on $400 \mathrm{kV}$ voltage level with length $200 \mathrm{~km}$ transmission line system .Extensive simulation test results show the algorithm correctly detects faults in each line section. The algorithm has been tested for several fault types symmetrical and unsymmetrical. The relay signal output $1 / 0$ of the simulator during normal operation condition the relay signal to circuit break is 1 the breakers it is closed, during symmetrical or unsymmetrical fault the relay signal to circuit break is 0 the breakers it is opened. The developed algorithm is accurate and very fast in detecting abnormal conditions in the system and this improves the reliability and stability of overall system.

\section{REFERENCES}

[1] A. Wright and C. Christopoulos, Electrical Power System Protection, Chapman \& Hall, London, U.K, 1993.

[2] L.V. Bewley, "Traveling waves on transmission systems", Wiley, New York, 1951.

[3] Blackburn, J. Lewis, Protective Relaying-Principles and Applications, Marcel Dekker Inc., New York and Basel, 1987.

[4] Areva T\&D, Network Protection and Automation Guide (NPAG), 1987.

[5] H. Lee, A. M. Mousa, GPS Traveling Wave Fault Locator Systems: Investigation into the Anamalous Measurements Related to Lightning Strikes, IEEE Transactions on Power Delivery, vol. 2, n.3, 1996.

[6] L. V. Bewley, Traveling Waves on Transmission Systems, Dover Publications Inc., New York, Second Edition, 1963. 
[7] M. Chamia and S. Liberman, Ultra High Speed Relay for EHV/UHV Transmission Lines - Development, Design and Application, IEEE Transactions on Power Apparatus and Systems, Vol. PAS-97, No. 6, Nov/Dec 1978, pp. 2104- 2112.

[8] M. S. Sachdev (Coordinator), IEEE Tutorial Course Text: Computer Relaying, Publication No. 79 EH0148-7-PWR, 1979.

[9] M. S. Sachdev (Coordinator), IEEE Tutorial Course: Advancements in Microprocessor Based Protection and Communication, IEEE Power Engineering Society, NJ, 1997.

[10] P.A. Crossley and P.G. McLaren, Distance Protection Based on Traveling Waves, IEEE Transactions on Power Apparatus and Systems, Vol. PAS-102, No. 9, September 1983, pp. 2971-2978.

[11] Juan M.Gers, Edward J.Holmes -Protection of Electricity Distribution Networks-2nd Edition -Institution of Engineering and Technology-2005

[12] Gerhard Ziegler, Numerical Distance Protection, Public is Corporate Publishing, Erlangen, Siemens, second edition, 2006, ISBN389578 2661.

[13] Michael Faraday House- Six Hills Way, Stevenage Power System Protection -Institution of Electrical Engineers-2005

[14] J. Duncan Glover, Mulukutlas. Sarma, Thomas J.Overbye- Power System Analysis And Design-Fifth Edition- Publisher, Global Engineering-2012,2008 Cengage Learning

\section{AUTHOR's BIOGRAPHY}

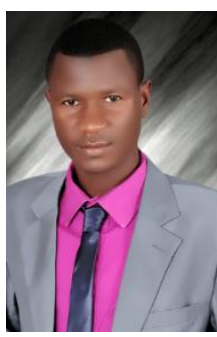

Modawy Adam Ali Abdalla, born in Elfasher-Sudan in 1987. Obtained his BSc. in Electrical and Electronic Engineering (Power) at Nyala University. He Received his MSc. in Electrical Engineering (Power) from Sudan University of Science and Technology. Lecturer and head Department of Electrical and Electronic Engineering, Faculty of Engineering Sciences, Nyala University. His field interest is in Renewable Energy, Power System Protection, and Machines and Devices.

Citation: Modawy Adam Ali Abdalla et al.(2017). Design of Travelling Wave Relay for Protection of Transmission Lines, International Journal of Research Studies in Electrical and Electronics Engineering (IJRSEEE), 3(4), pp.29-41, DOI: http://dx.doi.org/10.20431/2454-9436.0304005.

Copyright: (C) 2017 Modawy Adam Ali Abdalla et al. This is an open-access article distributed under the terms of the Creative Commons Attribution License, which permits unrestricted use, distribution, and reproduction in any medium, provided the original author and source are credited 